\title{
NON-ANNIHILATION OF TRAVELLING PULSES IN A REACTION-DIFFUSION SYSTEM*
}

\author{
M. MIMURA $^{\dagger}$, M. NAGAYAMA ${ }^{\ddagger}$, AND T. OHTA ${ }^{\S}$
}

\begin{abstract}
It is demonstrated that slowly travelling pulses arising in a reaction-diffusion(RD) system with the FitzHugh-Nagumo type nonlinearity do not necessarily annihilate but reflect off of each other before they collide. This phenomenon is in contrast with the well-known annihilation of travelling pulses on nerve axon and expanding rings in the Belousov-Zhabotinsky chemical reaction. By using singular perturbation methods, we derive a fourth order system of ODEs from the RD system, and study non-annihilation phenomenon of very slowly travelling pulses.
\end{abstract}

1. Introduction. It is well known that excitable pulses propagating on nerve axon or expanding rings in the 2-dimensional Belousov-Zhabotinsky chemical reaction annihilate one another on collision. It had been believed that this phenomenon is a typical feature of mono-stable excitable reaction-diffusion (RD) systems([18], for instance), from pulse-dynamics viewpoint. Alternatively, the non-annihilation phenomenon of travelling pulses has been recently observed in some excitable reactiondiffusion systems $([8],[10],[15],[16])$. As an example, Fig.1.1 demonstrates repulsive interaction of two slowly travelling pluses in the RD system proposed by Gray and Scott $[2]$

$$
\left\{\begin{array}{l}
u_{t}=u_{x x}+\varepsilon^{-1}\left(-a u+u^{2} v\right), \\
v_{t}=d v_{x x}+h(1-v)-u^{2} v
\end{array} \quad t>0, \quad x \in \mathbf{R}^{n},\right.
$$

where $a, h, d$ and $\varepsilon$ are suitable positive constants.

We conjectured in [11] that travelling pulses arising in more general RD systems possibly reflect each other before collision, if the velocity is very slow. The reason can be intuitively stated as follows: when two slowly travelling pulses approach closely, the concentration of the reactant $v$ between two pulses is gradually lower than the one in the back part of each pulse so that there occur two possibilities: two pulses fade out before collision or repel each other and move to the reversed direction, recovering their original shapes.

However, theoretical understanding of such non-annihilation of travelling pulses has not yet been established. There are mainly two difficulties : (i) Such nonannihilation is not always observed but occurs only in some restricted parameter regime in the system, in other words, annihilation or non-annihilation depends sensitively on values of parameters. (ii) this behavior is really a transient process.

In order to overcome these difficulties, we pick up a specific RD system with a small parameter $\varepsilon$ (see (2.1)), and take the singular limit procedure as $\varepsilon \downarrow 0$, which enables to trace the location of travelling pulses. Moreover, assuming that the velocity is very slow, we obtain a system of ODEs which describes the front and back interfacial points of pulses, and discuss this system to show the non-annihilation behavior of slowly travelling pulses.

\footnotetext{
${ }^{*}$ Received July 24, 2001; accepted for publication December 13, 2002.

$\dagger$ Department of Mathematical and Life Sciences, Hiroshima University, 1-3-1 Kagamiyama, Higashi-Hiroshima, 739-8526, Japan (mimura@math.sci.hiroshima-u.ac.jp).

${ }^{\ddagger}$ Research Institute for Mathematical Sciences, Kyoto University, Kitashirakawa, Sakyo-Ku, Kyoto, 606-8502, Japan (nagayama@kurims.kyoto-u.ac.jp).

$\S$ Department of Mathematical and Life Sciences, Hiroshima University, 1-3-1 Kagamiyama, Higashi-Hiroshima, 739-8526, Japan (ohta@math.sci.hiroshima-u.ac.jp).
} 


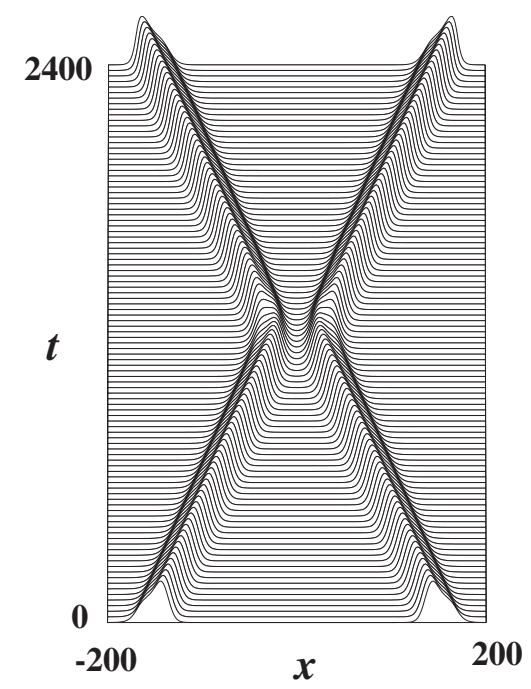

FiG. 1.1. Repulsion of two travelling pulses of (1.1) where $a=0.07, h=0.018, \varepsilon=1.0$.

2. Bistable reaction-diffusion system. The system which we treat is the following 1-dimensional FitzHugh-Nagumo(FHN) system with a sufficiently small parameter $\varepsilon>0[12]$ :

$$
\left\{\begin{array}{l}
\varepsilon \tau \frac{\partial u}{\partial t}=\varepsilon^{2} \frac{\partial^{2} u}{\partial x^{2}}+f(u)-v, \\
\frac{\partial v}{\partial t}=d \frac{\partial^{2} v}{\partial x^{2}}+u-\gamma v
\end{array} \quad t>0, \quad x \in \mathbf{R}\right.
$$

with $f(u)=u(1-u)(u-a)$, where $\varepsilon, \tau, d, a$ and $\gamma$ are positive constants and particularly, $\varepsilon$ is sufficiently small and $0<a<1 / 2$. We assume that $f(u)-v=0$ and $u-\gamma v=0$ have three intersection points, say $P=(0,0), Q=\left(u_{Q}, v_{Q}\right)$ and $R=\left(u_{R}, v_{R}\right)$, as in Fig.2.1 where $P$ and $R$ are stable constant equilibrium points of (2.1), while $Q$ is an unstable one. We impose the boundary conditions to (2.1) as

$$
(u, v)(t, \pm \infty)=P .
$$

Let $\gamma=\gamma_{*}$ be given such that $f(u)-v=0$ and $u-\gamma_{*} v=0$ have an odd symmetry with respect to the point $Q$ in the $(u, v)$-plane. Assuming that $\gamma$ is chosen to be less than $\gamma_{*}$, it is shown that (2.1) with (2.2) has travelling pulse solutions with one front and one back layers in $u$ for relatively small $\tau$ [7]. If $\gamma$ is close to $\gamma_{*}$ so that $f(u)-v=0$ and $u-\gamma v=0$ take nearly odd symmetry with respect to $Q$, the distance between two layers is rather long and when $\gamma$ tends to $\gamma_{*}$, it becomes infinity so that the pulse tends to either a front wave or a back one [5].

When $\gamma$ is close to $\gamma_{*}$, we consider the interaction of two travelling pulses. When $\tau$ is small, the travelling pulses annihilate on collision, as in Fig.2.2. As $\tau$ increases, these pulses move slowly and fade out before collision, as in Fig.2.3 and as $\tau$ increases slightly further, the pulses move more slowly and they repel each other in such a way that two facing travelling front layers repel each other twice, and each pair of front and back layers approach and repel each other once, as in Fig.2.4. Although the 


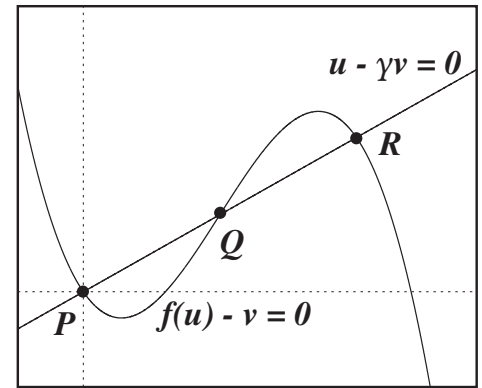

FIG. 2.1. Nulclines of $f$ and $g$ in (2.1) where $a=0.25, \gamma=10.2857$.

repulsive behavior in Fig.2.4 is qualitatively different from the one in the RD system (1.1) in Fig.1.1, it could be confirmed that very slowly travelling pulse solutions of (2.1) with (2.2) possess non-annihilation property.

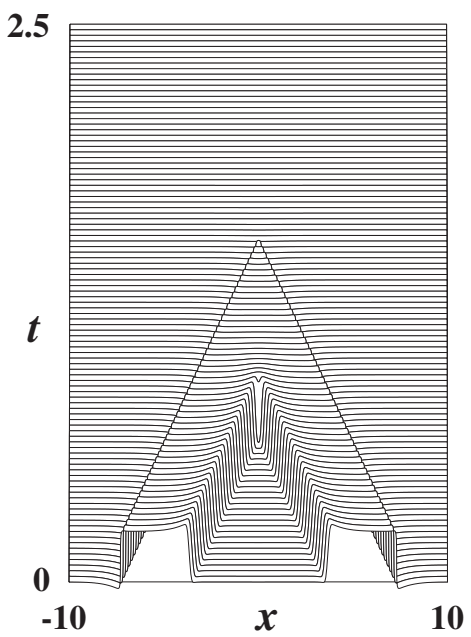

FIG. 2.2. Annihilation of two travelling pulses of (2.1) where $d=1.0, a=0.25, \gamma=10.2857, \tau=$ $0.035, \varepsilon=0.025$.

3. Limiting system as $\varepsilon \downarrow 0$. We consider a pulse-like solution with one front and one back layer of the bistable FHN system (2.1) with (2.2) in R. By taking the limit as $\varepsilon \downarrow 0$, each layer becomes the corresponding interface as in Fig.3.1. Let $\eta_{F}(t)$ and $\eta_{B}(t)$ satisfying $0<\eta_{F}(t)<\eta_{B}(t)$ be respectively the front and back interfaces. The singular perturbation methods ([1], for instance) lead the evolutional equations of $\eta_{F}(t)$ and $\eta_{B}(t)$ which are described by

$$
\begin{gathered}
\tau \dot{\eta_{F}}(t)=-\lambda\left(v\left(t, \eta_{F}(t)\right)\right), \quad t>0, \\
\tau \dot{\eta_{B}}(t)=\lambda\left(v\left(t, \eta_{B}(t)\right)\right), \quad t>0,
\end{gathered}
$$

where dot means the first derivative of time $t$. For each number $\xi$ such that $f(u)-\xi=0$ has three zeros $u=h_{ \pm}(\xi)$ and $h_{0}(\xi)$, as in Fig.3.2, $\lambda(\xi)$ is the velocity of a travelling 


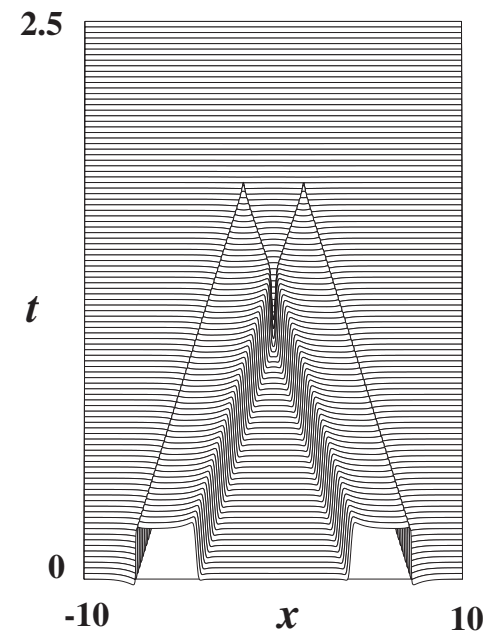

FIG. 2.3. Annihilation of two travelling pulses of (2.1) where the parameters are the same as those in Fig.2.2 except $\tau=0.04311$.

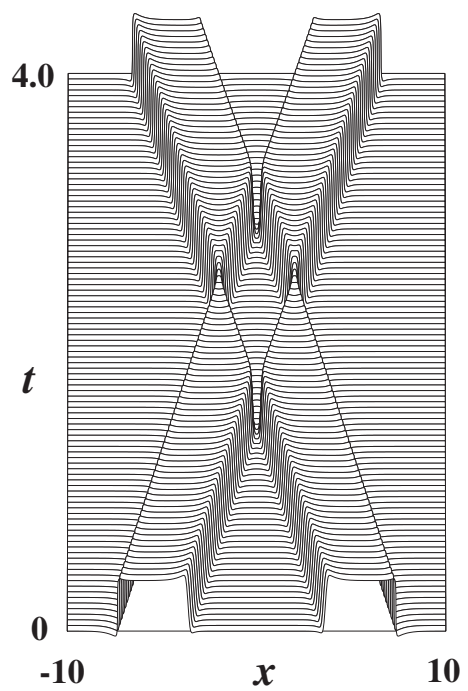

FIG. 2.4. Repulsion of two travelling pulses of (2.1) where the parameters are the same as those in Fig.2.2 except $\tau=0.048$.

front solution $u(z)(z=x-\lambda t)$ of

$$
u_{t}=u_{x x}+f(u)-\xi, \quad z \in \mathbf{R}
$$

with the boundary conditions

$$
\lim _{x \rightarrow \pm \infty} u(t, x)=h_{\mp}(\xi) .
$$


Here $\lambda(\xi)$ is uniquely determined for $\xi \in\left(v_{-}, v_{+}\right)$. By $(2.1)$, the equation for $v(t, x)$ is given by

$$
\frac{\partial v}{\partial t}=d \frac{\partial^{2} v}{\partial x^{2}}+\left\{\begin{array}{lll}
h_{-}(v)-\gamma v, & t>0, & x \in \mathbf{R} \backslash\left(\eta_{F}(t), \eta_{B}(t)\right) \\
h_{+}(v)-\gamma v, & t>0, & x \in\left(\eta_{F}(t), \eta_{B}(t)\right)
\end{array}\right.
$$

with

$$
v(t, \cdot) \in C^{1}(\mathbf{R}), \quad t>0
$$

and

$$
\lim _{|x| \rightarrow \infty} v(t, x)=0, \quad t>0 .
$$

The derivation of (3.1)-(3.7) is stated in Appendix A. It turns out that (3.1)-(3.7) is a free boundary problem for the free boundaries $\eta_{F}(t)$ and $\eta_{B}(t)$.

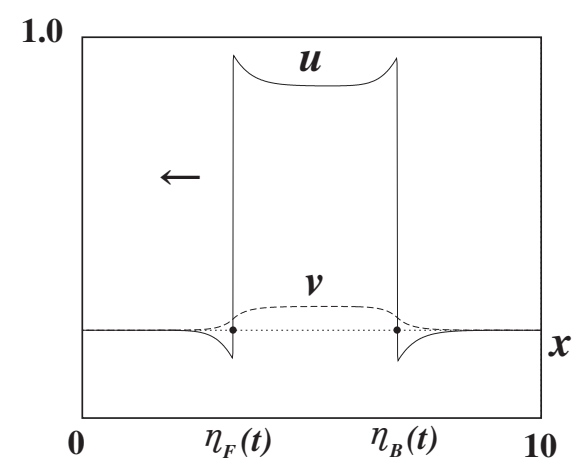

FIG. 3.1. A travelling pulse of (2.1) with $\varepsilon \downarrow 0$ where the parameters are the same as those in Fig.2.2 expect $\tau=0.04685$.

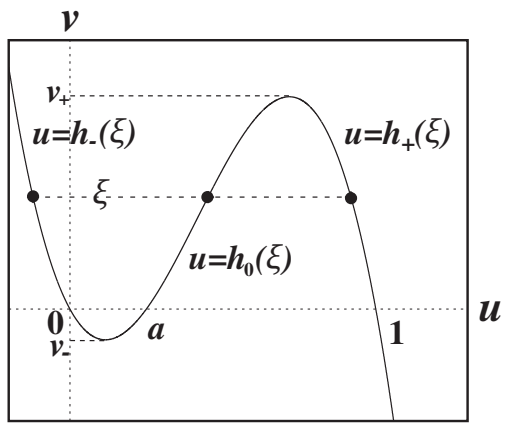

FIG. 3.2. Graph of the equation $f(u)-v=0$, where $u=h_{-}(v), u=h_{+}(v)$ and $u=h_{0}(v)$ denote three branches of $f(u)-v=0$.

A travelling pulse solution of (3.1)-(3.7) can be described by $v(t, x)=V(z) \quad(z=$ $x-c t$ ) with constant velocity $c$, coupled with the interfaces $\eta_{F}(t)=z_{F}=x_{F}-c t$ and $\eta_{B}(t)=z_{B}=x_{B}-c t$ (Fig.3.3). The resulting problem for $(V(z), c)$ with unknowns $z_{F}$ and $z_{B}$ is stated as follows:

$$
-\tau c=-\lambda\left(V\left(z_{F}\right)\right)=\lambda\left(V\left(z_{B}\right)\right),
$$




$$
\begin{gathered}
-c V_{z}=d V_{z z}+\left\{\begin{array}{cl}
h_{-}(V)-\gamma V, & z \in \mathbf{R} \backslash\left(z_{F}, z_{B}\right), \\
h_{+}(V)-\gamma V, & z \in\left(z_{F}, z_{B}\right),
\end{array}\right. \\
V \in C^{1}(\mathbf{R}), \\
\lim _{|z| \rightarrow \infty} V(z)=0 .
\end{gathered}
$$

Ikeda[4] proved the existence of travelling and standing pulse solutions of (3.8)-(3.11). For any $\tau$, there is uniquely a standing pulse solution of (3.8)-(3.11). Furthermore, there is the critical value $\tau_{c}$ such that there are travelling pulses $V(z ; \tau)$ with velocity $\pm c(\tau)$ for $0<\tau<\tau_{c}$. Furthermore, he showed that these are super-critically bifurcated from the standing pulse.

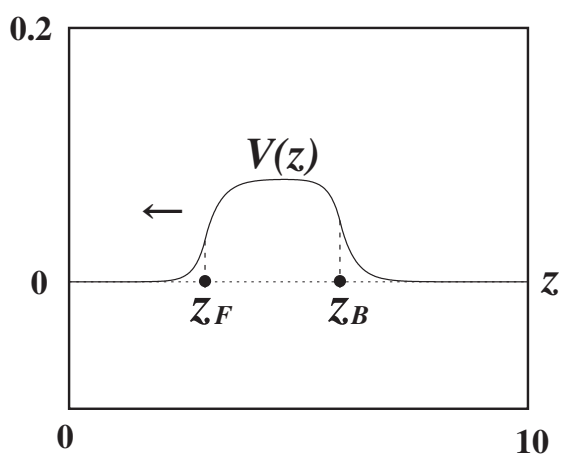

FIG. 3.3. A travelling pulse solution $V(z)$ of (3.8)-(3.11) where $d=1.0, a=0.25, \gamma=$ $10.28571, \tau=0.044$

4. Pulse-pulse interaction in the limit system. In this section, we consider the repulsive interaction of two travelling pulses of (3.8)-(3.11) for fixed $\tau>0$, where they are located symmetrically with $x=0$ in $\mathbf{R}$. In order to analyze this interaction, we assume that there is the reflecting wall at $x=0$ and that one of the pulses moves to the left direction in $\mathbf{R}_{+}=(0,+\infty)$. Let $x=\eta_{1}(t), x=\eta_{2}(t)$ be interfaces satisfying $0<\eta_{2}(t)<\eta_{1}(t)<+\infty$ where $2 \eta_{2}(t)$ is the distance between two front interfaces. The resulting problem of (3.1)-(3.7) can be stated as follows:

$$
\begin{gathered}
\tau \dot{\eta_{1}}=\lambda\left(v\left(t, \eta_{1}(t)\right)\right), \quad t>0 \\
\tau \dot{\eta_{2}}=-\lambda\left(v\left(t, \eta_{2}(t)\right)\right), \quad t>0 \\
\frac{\partial v}{\partial t}=d \frac{\partial^{2} v}{\partial x^{2}}+\left\{\begin{array}{l}
h_{-}(v)-\gamma v, \quad t>0, x \in \mathbf{R}_{+} \backslash\left(\eta_{2}(t), \eta_{1}(t)\right), \\
h_{+}(v)-\gamma v, \quad t>0, x \in\left(\eta_{2}(t), \eta_{1}(t)\right),
\end{array}\right. \\
v(t, \cdot) \in C^{1}\left(\mathbf{R}_{+}\right)
\end{gathered}
$$

with the boundary and initial conditions

$$
\frac{\partial v}{\partial x}=0, \quad t>0, \quad x=0,
$$




$$
\lim _{x \rightarrow+\infty} v=0, \quad t>0
$$

and

$$
\left\{\begin{array}{l}
\eta_{1}(0)=\eta_{10} \\
\eta_{2}(0)=\eta_{20}, \\
v(0, x)=v_{0}(x), \quad x \in \mathbf{R}_{+} .
\end{array}\right.
$$

The existence and uniqueness of solutions to (4.1)-(4.7) are proved in [3] and the convergence between the RD system (2.1) and ones of the limiting system (4.1)-(4.7) as $\varepsilon \downarrow 0$ is discussed in [19].

Now, our problem can be formulated as follows: Suppose that the initial condition $\left(v_{0}(x), \eta_{10}, \eta_{20}\right)$ are chosen to approximately satisfy a slowly travelling pulse $\left(V(x ; \tau), x_{B}, x_{F}\right)$ for large $x_{B}$ and $x_{F}$. When $\tau$ is suitably fixed to generate that $c(\tau)$ is very small, does the solution $\left(v(t, x), \eta_{1}(t), \eta_{2}(t)\right)$ of (4.1)-(4.7) exhibit repulsive behavior when it approaches the wall $x=0$ ?

We rely on numerical procedures to solve (4.1)-(4.7) which is a free boundary problem for $\eta_{1}(t)$ and $\eta_{2}(t)$. When $\tau$ is very small, these travelling interfaces annihilate after collision, as in Fig.4.1. When $\tau$ increases, two travelling interfaces annihilate before collision, as in Fig.4.2, when $\tau$ is still increases but less than $\tau_{c}$, these interfaces reflect each other, as in Fig.4.3. Comparing Figs.4.1-4.3 and Figs.2.2-2.4, one finds that the limiting problem (4.1)-(4.7) is qualitatively a good approximation to the bistable FHN system (2.1) with (2.2) with sufficiently small $\varepsilon>0$, from the pulsepulse interaction view point.

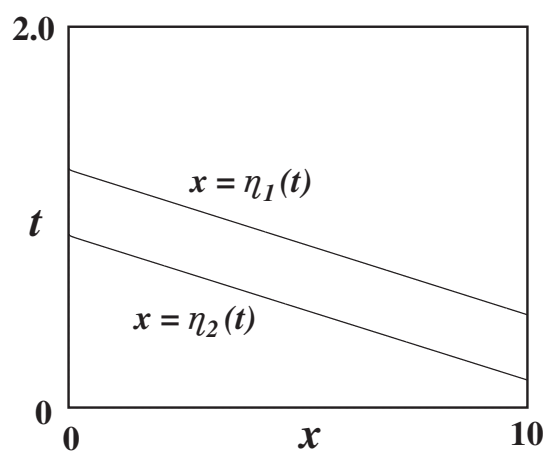

FiG. 4.1. Annihilation of travelling pulses of (4.1)-(4.7) where $d=1.0, a=0.25, \gamma=$ $10.28571, \tau=0.034$.

\section{Equations of interface-interface interaction.}

5.1. Existence of travelling pulses. As was shown in Fig.4.1, the reflection process of interfaces is truly transient. In order to study this problem, we further reduce (4.1)-(4.7) to a system of ODEs for the interfaces $\eta_{1}(t)$ and $\eta_{2}(t)$ only, assuming that they move very slowly.

For this purpose, we approximate $f(u)$ in $(2.1)$ by a piecewise linear function $f(u)=-u+H(u-a)$ with a constant satisfying $0<a<1 / 2$ where $H(s)=1$ for $s>0$ and $H(s)=0$ for $s<0$ (see Fig.5.1). Then the velocity $\lambda(\xi)$ of (3.3) is explicitly 


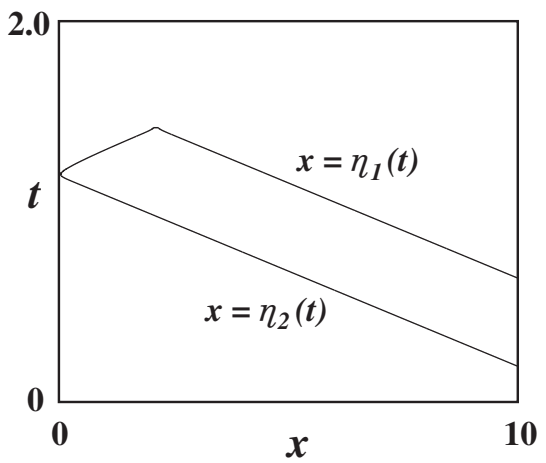

FIG. 4.2. Annihilation of travelling pulses of (4.1)-(4.7) where the parameters are the same as those in Fig.4.1 except $\tau=0.0375$.

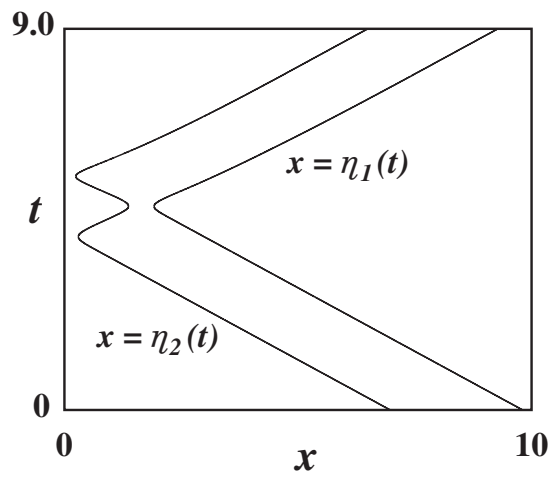

FIG. 4.3. Repulsion of travelling pulses of (4.1)-(4.7) where the parameters are the same as those in Fig.4.1 except $\tau=0.044$.

given as

$$
\lambda(\xi)=\frac{1-2 a-2 \xi}{\sqrt{(a+\xi)(-1+a+\xi)}}
$$

[17] or equivalently

$$
\frac{\lambda(\xi)}{\sqrt{\lambda^{2}(\xi)+4}}=1-2 a-2 \xi
$$

With $z=x-c t$, travelling pulse solutions $V(z)$ with velocity $c$ are obtained by solving the following problem resulting from (3.8)-(3.11):

$$
\begin{aligned}
& \frac{\tau c}{\sqrt{(\tau c)^{2}+4}}=1-2 a-V\left(z_{F}\right) \\
& \frac{\tau c}{\sqrt{(\tau c)^{2}+4}}=-1+2 a+V\left(z_{B}\right)
\end{aligned}
$$




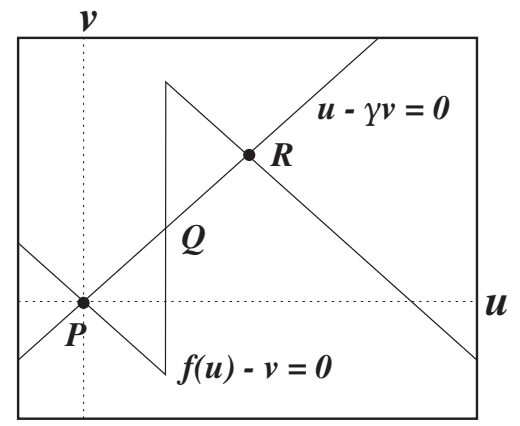

FIG. 5.1. Nulclines of $f$ and $g$ of the piecewise-linear nonlinearities in (2.1) where $a=0.25, \gamma=$ 0.9999999 .

and

$$
\begin{gathered}
-c V_{z}=d V_{z z}+ \begin{cases}-(1+\gamma) V, & x \in \mathbf{R} \backslash\left(z_{F}, z_{B}\right), \\
-(1+\gamma) V+1, & x \in\left(z_{F}, z_{B}\right),\end{cases} \\
V \in C^{1}(\mathbf{R}), \\
\lim _{|z| \rightarrow \infty} V(z)=0 .
\end{gathered}
$$

For fixed $c$ and $z_{B}-z_{F}=s$, we obtain the unknown $V$ by (5.5)-(5.7). Substituting it into (5.3) and (5.4), we have the following equations for $c$ and $s$ :

$$
\begin{aligned}
& \frac{\tau c}{\sqrt{(\tau c)^{2}+4}}=1-2 a-\frac{\phi_{-}}{\beta \phi}\left(1-\exp \left(-\frac{\phi_{+}}{2 d} s\right)\right), \\
& \frac{\tau c}{\sqrt{(\tau c)^{2}+4}}=-1+2 a+\frac{\phi_{+}}{\beta \phi}\left(1-\exp \left(-\frac{\phi_{-}}{2 d} s\right)\right),
\end{aligned}
$$

where $\beta=1+\gamma, \phi(c)=\sqrt{c^{2}+4 d \beta}$ and $\phi_{ \pm}(c)=\phi(c) \pm c$. It is convenient to rewrite $(5.8),(5.9)$ as

$$
\begin{gathered}
F(c, s)=\frac{\phi_{-}}{2 \beta \phi} \exp \left(-\frac{\phi_{+}}{2 d} s\right)+\frac{\phi_{+}}{2 \beta \phi} \exp \left(-\frac{\phi_{-}}{2 d} s\right)-2 a-\frac{1}{\beta}+1=0, \\
G(c, s ; \tau)=\frac{\tau c}{\sqrt{(\tau c)^{2}+4}}-\frac{c}{\beta \phi}-\frac{\phi_{-}}{2 \beta \phi} \exp \left(-\frac{\phi_{+}}{2 d} s\right)+\frac{\phi_{+}}{2 \beta \phi} \exp \left(-\frac{\phi_{-}}{2 d} s\right)=0 .
\end{gathered}
$$

When $2 a+1 / \beta-1=0$ (or equivalently $\gamma=\gamma_{*}=2 a /(1-2 a)$ ), $f(u)-v=0$ and $u-\gamma_{*} v=0$ take odd symmetry. When $2 a+1 / \beta-1 \leq 0$ (or $\gamma \geq \gamma_{*}$ ), one knows $F(c, s)>0$ so that there is no travelling solution. We therefore assume $2 a+1 / \beta-1>0$ (or $\left.0<\gamma<\gamma_{*}\right)$. It is obvious that $(c, s)=\left(0, s_{0}\right)$ with

$$
s_{0}=-\sqrt{d / \beta} \log (2 a \beta+1-\beta)
$$


is a solution of (5.10) and (5.11) for any $\tau>0$. This yields a standing pulse solution of (5.1)-(5.7). Taking $\tau$ as a free parameter and keeping $a, \gamma\left(<\gamma_{*}\right)$ and $d$ to be suitably fixed, we find that there is a critical value $\tau_{c T}$ such that travelling pulse solutions of (5.1)-(5.7) exist for $\tau<\tau_{c T}$, which are bifurcated from the standing one at $\tau=\tau_{c T}$ (Fig.5.2). The relation between the parameter $\tau$ and the width $s$ is shown in Fig.5.3.

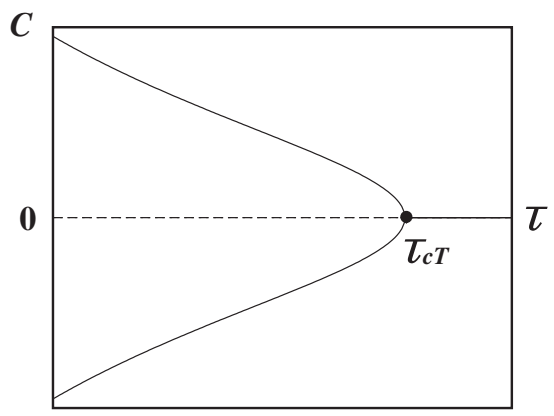

FIG. 5.2. Relation between the velocity $c$ and the parameter $\tau$ where $d=1.0, a=0.25, \gamma=$ $0.9999999, \tau_{c t}=0.353554$.

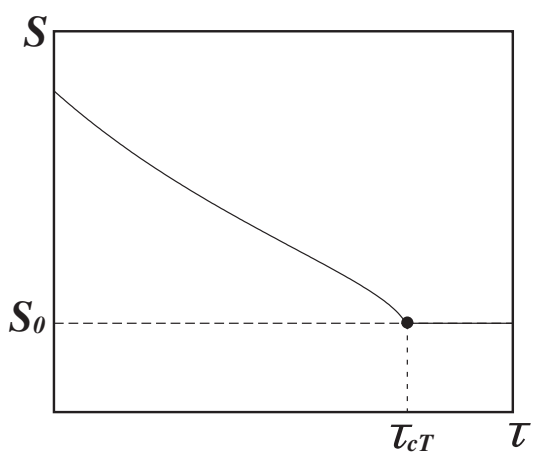

FIG. 5.3. Relation between the width $s$ and the parameter $\tau$ where the parameters are the same as those in Fig.5.2.

If $\tau$ is chosen to be near $\tau_{c T}$, the velocity $c$ is very small so that its explicit form can be obtained. Using $F\left(0, s_{0}\right)=0$ and $\frac{\partial F}{\partial s}\left(0, s_{0}\right) \neq 0$, the implicit function theorem says that $F(c, s)=0$ has a solution $s=h(c)$ in a neighborhood of $c=0$ satisfying $s_{0}=h(0)$. It is expanded in terms of $c$ up to $O\left(c^{2}\right)$ as follows:

$$
s=h(c)=s_{0}+s_{1} c^{2},
$$

where

$$
s_{1}=\frac{1}{16}\left(\frac{s_{0}}{d \beta}+\frac{s_{0}^{2}}{d \sqrt{d \beta}}\right)
$$


Substituting (5.13) into $G(c, s ; \tau)=0$ and expanding it with small $c$, we obtain the following relation up to $O\left(c^{3}\right)$ :

$$
\frac{c}{2}\left(\tau-\tau_{*}\right)+g(\tau) c^{3}=0
$$

where

$$
\tau_{*}=\frac{1}{\beta \sqrt{d \beta}}\left(1-\left(1+s_{0} \sqrt{\beta / d}\right) \exp \left(-s_{0} \sqrt{\beta / d}\right)\right)>0
$$

and

$$
g(\tau)=\frac{1}{16}\left[\frac{1}{d \beta^{2} \sqrt{d \beta}}+\left(\frac{s_{0}^{3}}{3 d^{3} \beta}-\frac{s_{0}}{d^{2} \beta^{3}}-\frac{1}{d \beta^{2} \sqrt{d \beta}}-\frac{8 s_{0} s_{1}}{d \sqrt{d \beta}}\right) \exp \left(-\sqrt{\beta / d} s_{0}\right)-\tau^{3}\right] .
$$

When $\gamma$ is near $\gamma_{*}$ and $\tau$ is also near $\tau_{*}$, one finds $g(\tau)>0$ so that the velocity $c$ is expressed as $c= \pm \sqrt{\left(\tau_{*}-\tau\right) / 2 g(\tau)}$.

5.2. Stability of travelling pulse solutions. We now consider the stability of travelling pulse solutions in $\boldsymbol{R}$. The spectral analysis shows that when $\tau$ decreases, the standing pulse solution of (3.1)-(3.7) is primarily destabilized through Hopf bifurcation at $\tau=\tau_{c O}$ and then secondly through translational one at $\tau=\tau_{c T} \quad\left(\tau_{c T}<\tau_{c O}\right)$ [6], where the bifurcating travelling pulse solutions globally exist for $\tau<\tau_{c T}$. This information tells us that they are unstable when $\tau$ is near $\tau_{c T}$. However, there is the critical value, say $\tau=\tau_{c O T}\left(\tau_{c O T}<\tau_{c T}\right)$ so that the unstable travelling pulse solutions recover their stability for $\tau<\tau_{c O T}$ (Fig.5.4) [7]. We should note that if $\gamma$ is less than but near $\gamma_{*}$ (i.e. $f(u)-v=0$ and $u-\gamma v=0$ take nearly odd symmetry), three critical values $\tau_{c O T}, \tau_{c T}$ and $\tau_{c O}$ are very close with each other (in fact, if they are completely odd symmetric, then $\tau_{c O T}=\tau_{c T}=\tau_{c O}$ hold). We thus find that there exist very slowly travelling pulse solutions which are stable, if $\gamma$ is near $\gamma_{*}$ and $\tau$ is also near $\tau_{c O T}$.

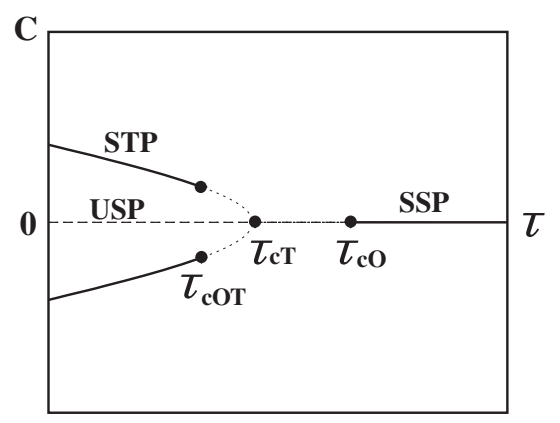

FIG. 5.4. Schematic bifurcation diagram of travelling pulses with the parameter $\tau$, where the vertical axis indicates the velocity of the travelling pulse. (SSP: stable standing pulse, USP: unstable standing pulse, STP: stable travelling pulse.)

5.3. A system describing pulse-pulse interaction. Consider the situation where there is a pulse-like solution $\left(v(t, x), \eta_{1}(t), \eta_{2}(t)\right) \quad\left(\eta_{2}(t)<\eta_{1}(t)\right)$ in $\boldsymbol{R}_{+}$with the reflecting wall $x=0$. The problem resulting from (4.1)-(4.7) with (5.1) is written as

$$
\frac{\tau \dot{\eta_{1}}(t)}{\sqrt{\left(\tau \dot{\eta_{1}}(t)\right)^{2}+4}}=1-2 a-2 v\left(t, \eta_{1}(t)\right), \quad t>0,
$$




$$
\frac{\tau \dot{\eta_{2}}(t)}{\sqrt{\left(\tau \dot{\eta_{2}}(t)\right)^{2}+4}}=-1+2 a+2 v\left(t, \eta_{2}(t)\right), \quad t>0
$$

where $\eta_{2}(t)<\eta_{1}(t)$ is assumed for any $t>0$, and

$$
\begin{gathered}
\frac{\partial v}{\partial t}=d \frac{\partial^{2} v}{\partial x^{2}}+ \begin{cases}-\beta v, & x \in \mathbf{R}_{+} \backslash\left(\eta_{2}(t), \eta_{1}(t)\right) \\
-\beta v+1, & \left.x \in\left(\eta_{2}(t), \eta_{1}(t)\right)\right)\end{cases} \\
v(t, \cdot) \in C^{1}\left(\mathbf{R}_{+}\right) \\
\frac{\partial v}{\partial x}=0, \quad x=0, \quad t>0 \\
\lim _{x \rightarrow \infty} v=0, \quad t>0 \\
\left\{\begin{array}{l}
\eta_{1}(0)=\eta_{10}, \\
\eta_{2}(0)=\eta_{20}\left(<\eta_{10}\right) \\
v(0, x)=v_{0}(x), \quad x \in \mathbf{R}_{+}
\end{array}\right.
\end{gathered}
$$

By using the advantage of piecewise-linear nonlinearity of (5.3) in (5.20), $v\left(t, \eta_{i}(t)\right)(i=1,2)$ can be determined for given $\eta_{i}(t)(i=1,2)$. We thus formally obtain the closed system for $\eta_{1}(t)$ and $\eta_{2}(t)$, which is described by integral equations with respect to time as well as space. To obtain this system, we employ the following formal approximation:

$$
\eta_{i}(\tilde{t})=\eta_{i}(t)+(\tilde{t}-t) \dot{\eta}_{i}(t)+\frac{(\tilde{t}-t)^{2}}{2} \ddot{\eta}_{i}(t)+\cdots
$$

which is valid for sufficiently weak variation of $\eta_{i}(i=1,2)$. It is shown in Appendix $\mathrm{C}$ that the expansion in (5.25) is actually an expansion in powers of $\delta=\sqrt{\tau_{*}-\tau}$. Therefore, the truncation up to the second derivative with respect to time is justified near the bifurcation threshold $\tau=\tau_{*}$.

After straightforward calculation in (5.18)-(5.24), we obtain the following fourth order system of ODEs for $\eta_{1}(t)$ and $\eta_{2}(t)$ up to $O\left(\ddot{\eta}_{i}\right)(i=1,2)$ :

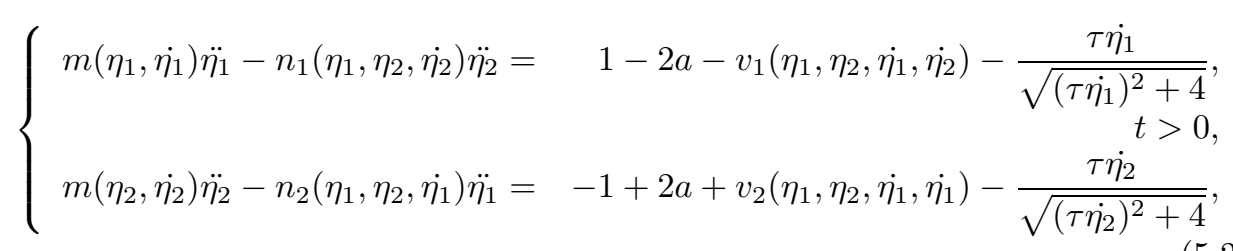

where the coefficient functions $m, n_{1}, n_{2}$ are non-negative for all $\eta_{1}, \eta_{2}, \dot{\eta}_{1}, \dot{\eta}_{2}$, and $v_{1}, v_{2}$ describe the interaction term between the two travelling pulses. The derivation of (5.26), the forms of functions $m, n_{1}, n_{2}, v_{1}$ and $v_{2}$ are explicitly stated in Appendix B.

Suppose that $\eta_{1}(t)-\eta_{2}(t)$ is finite and $\eta_{1}(t)$ is very large for any $t>0$. Then we find by (B.7), (B.9), (B.13)-(B.15) that (5.26) is reduced to the following slightly 
simplified fourth order system of ODEs:

$$
\left\{\begin{array}{rr}
\bar{m}\left(\dot{\eta}_{1}\right) \ddot{\eta_{1}}-\tilde{n}\left(\eta_{1}-\eta_{2}, \dot{\eta_{2}}\right) \ddot{\eta}_{2}= & 1-2 a-\tilde{v}_{1}\left(\eta_{1}-\eta_{2}, \dot{\eta_{1}}, \dot{\eta_{2}}\right)-\frac{\tau \dot{\eta_{1}}}{\sqrt{\left(\tau \dot{\left.\eta_{1}\right)^{2}+4}\right.}}, t>0, \\
m\left(\eta_{2}, \dot{\eta_{2}}\right) \ddot{\eta_{2}}-\tilde{n}\left(\eta_{1}-\eta_{2},-\dot{\eta_{1}}\right) \ddot{\eta_{1}}= & -1+2 a+\tilde{v}_{2}\left(\eta_{1}, \eta_{2}, \dot{\eta_{1}}, \dot{\eta_{2}}\right)-\frac{\tau \dot{\eta_{2}}}{\sqrt{\left(\tau \dot{\left.\eta_{2}\right)^{2}+4}\right.}}
\end{array}\right.
$$

where

$$
\begin{gathered}
\bar{m}(k)=\frac{12 d^{2}}{\phi(k)^{5}} \\
\tilde{n}(s, k)=\left(\frac{s^{2}}{\phi^{3}(k)}+\frac{6 d s}{\phi^{4}(k)}+\frac{12 d^{2}}{\phi^{5}(k)}\right) \exp \left(-s \frac{\phi_{+}(k)}{2 d}\right)
\end{gathered}
$$

with $\phi(k)=\sqrt{k^{2}+4 d \beta}$ and $\phi_{ \pm}(k)=\phi(k) \pm k$,

$$
\begin{gathered}
\tilde{v}_{1}\left(\eta_{1}-\eta_{2}, \dot{\eta}_{1}, \dot{\eta_{2}}\right)=\frac{\phi_{1-}}{\beta \phi_{1}}-\frac{\phi_{2-}}{\beta \phi_{2}} \exp \left(-\left(\eta_{1}-\eta_{2}\right) \frac{\phi_{2+}}{2 d}\right) \\
\tilde{v}_{2}\left(\eta_{1}, \eta_{2}, \dot{\eta}_{1}, \dot{\eta}_{2}\right)=\frac{\phi_{2+}}{\beta \phi_{2}}\left(1+\exp \left(-2 \eta_{2} \frac{\phi_{2-}}{2 d}\right)\right)-\frac{\phi_{1+}}{\beta \phi_{1}} \exp \left(-\left(\eta_{1}-\eta_{2}\right) \frac{\phi_{1-}}{2 d}\right)
\end{gathered}
$$

for $\phi_{i}=\phi\left(\dot{\eta}_{i}\right)=\sqrt{\dot{\eta}_{i}^{2}+4 d \beta}$ and $\phi_{i \pm}=\phi\left(\dot{\eta}_{i}\right) \pm \dot{\eta}_{i} \quad(i=1,2)$.

5.4. Reflection property of travelling pulses. We take the initial conditions as

$$
\left\{\begin{array}{l}
\eta_{1}(0)=\alpha+s(\tau) \\
\eta_{2}(0)=\alpha \\
\dot{\eta}_{i}(0)=-c(\tau)(<0) \quad(i=1,2)
\end{array}\right.
$$

where $\alpha$ is fixed arbitrarily large. Suppose that $s_{0}$ is very large, that is, $2 a+1 / \beta-1$ is positive but sufficiently small, and that $\tau$ is suitably fixed to require that $c(\tau)$ is very small and $s(\tau)$ is very close to $s_{0}$. Under this situation, numerical computation of (5.27) clearly shows that the front interface $\eta_{2}(t)$ reflects with the wall $x=0$ twice, while it reflect with the back $\eta_{1}(t)$ once, as in Fig.5.5. This behavior is qualitatively similar to the ones in Figs.4.3 and 2.4.

Now, let us analytically consider the above reflection-process demonstrated in Fig.5.5. We can classify the behavior of $\eta_{1}(t)$ and $\eta_{2}(t)$ into the following 3-stages:

(i) $\eta_{2}(t)$ reflects with the wall $x=0:\left(\eta_{1}(t) \gg 1, \eta_{1}(t)-\eta_{2}(t) \gg 1\right.$ and $\left.\dot{\eta}_{1}(t)<0\right)$;

(ii) $\eta_{1}(t)$ and $\eta_{2}(t)$ repel each other : $\left(\eta_{1}(t) \gg 1, \eta_{2}(t) \gg 1\right.$ and $\left.\eta_{1}(t)-\eta_{2}(t)=O(1)\right)$;

(iii) $\eta_{2}(t)$ reflects with the wall $x=0$ again : $\left(\eta_{1}(t) \gg 1, \eta_{1}(t)-\eta_{2}(t) \gg 1\right.$ and $\left.\dot{\eta}_{1}(t)>0\right)$.

Depending on the stages (i)-(iii), (5.27) can be simplified as follows:

Stage (i): Since $\eta_{1}(t)-\eta_{2}(t)$ is very large, one may expect that the interfaces $\eta_{1}(t)$ and $\eta_{2}(t)$ are independent, so that (5.27) is decoupled as

$$
\bar{m}\left(\dot{\eta}_{1}\right) \ddot{\eta}_{1}=1-2 a-\hat{v}_{1}\left(\dot{\eta}_{1}\right)-\frac{\tau \dot{\eta}_{1}}{\sqrt{\left(\tau \dot{\eta}_{1}\right)^{2}+4}}
$$




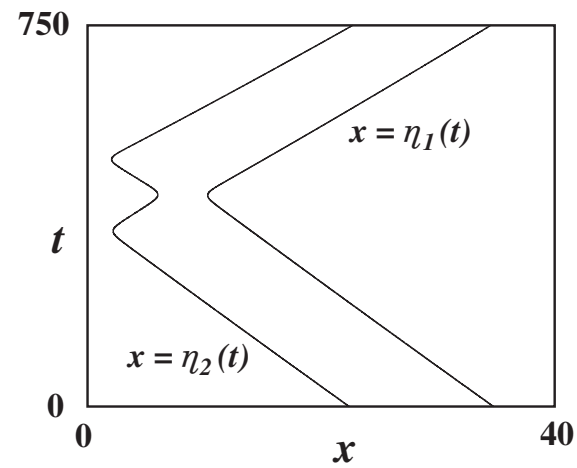

FIG. 5.5. Repulsion of the interfaces $\eta_{1}(t)$ and $\eta_{2}(t)$ where $d=1.0, a=0.25, \gamma=0.9999999, \tau=$ $0.3535, c=0.05669, s=12.1537$.

and

$$
m\left(\eta_{2}, \dot{\eta}_{2}\right) \ddot{\eta}_{2}=-1+2 a+\hat{v}_{2}\left(\eta_{2}, \dot{\eta}_{2}\right)-\frac{\tau \dot{\eta}_{2}}{\sqrt{\left(\tau \dot{\eta}_{2}\right)^{2}+4}}
$$

where

$$
\begin{gathered}
\hat{v}_{1}\left(\dot{\eta}_{1}\right)=\frac{\phi_{1-}}{\beta \phi_{1}} \\
\hat{v}_{2}\left(\eta_{2}, \dot{\eta}_{2}\right)=\frac{\phi_{2+}}{\beta \phi_{2}}\left(1+\exp \left(-2 \eta_{2} \frac{\phi_{2-}}{2 d}\right)\right) .
\end{gathered}
$$

Putting $\dot{\eta}_{1}=\xi_{1}$ into (5.33), we obtain the equation for $\xi_{1}$ only as

$$
\bar{m}\left(\xi_{1}\right) \dot{\xi}_{1}=1-2 a-\frac{1}{\beta}+\frac{\xi_{1}}{\beta \sqrt{\xi_{1}^{2}+4 d \beta}}-\frac{\tau \xi_{1}}{\sqrt{\left(\tau \xi_{1}\right)^{2}+4}} \equiv S\left(\xi_{1} ; \tau\right), \quad t>0 .
$$

As the functional form of $S\left(\xi_{1} ; \tau\right)$ is shown in Fig.5.6, there is the critical value $\tau_{c}$ such that (5.37) has one critical point $\xi_{1}=c_{1}<0$ for $\tau_{c}<\tau$ and three critical points $\xi_{1}=c_{i} \quad(i=1,2,3)$ satisfying $c_{1}<0<c_{2}<c_{3}$ for $0<\tau<\tau_{c}$. Noting that $\xi_{1}=c_{1}, c_{3}$ are asymptotically stable and $\xi_{1}=c_{2}$ is unstable, one finds that $\dot{\eta}_{1}(t)$ becomes asymptotically $c_{1}$, that is, $\eta_{1}(t)$ becomes a travelling interface with velocity $c_{1}$. Here we should remark that $c_{1}$ is the velocity of the travelling back solution $v(z) \quad(z=x-c t)$ of the following problem:

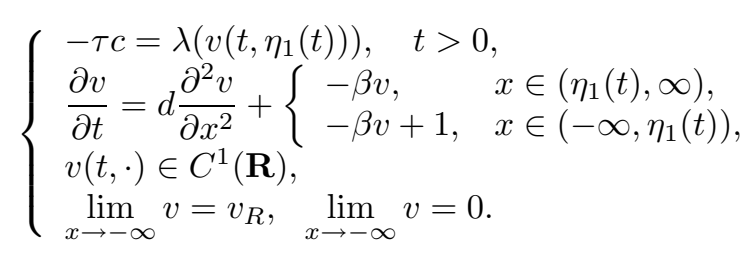

This indicates that the dynamics of the back interface $\eta_{1}(t)$ is approximated by the travelling back solution of (5.38). Next consider (5.34). If $\eta_{2}$ is very large, then $\xi_{2}=\dot{\eta_{2}}$ satisfies

$$
\bar{m}\left(\xi_{2}\right) \dot{\xi}_{2}=-1+2 a+\frac{1}{\beta}+\frac{\xi_{2}}{\beta \sqrt{\xi_{2}^{2}+4 d \beta}}-\frac{\tau \xi_{2}}{\sqrt{\left(\tau \xi_{2}\right)^{2}+4}} \equiv-S\left(-\xi_{2} ; \tau\right), \quad t>0 .
$$


Therefore, $\dot{\eta}_{2}(t)$ is approximately $-c_{3}$, that is, $\eta_{2}(t)$ becomes a travelling interface with velocity $-c_{3}$. However, if $\eta_{2}(t)$ approaches the wall $x=0$, that is, $\eta_{2}(t)=O(1)$, it is no longer described by (5.39) only but by (5.34). We conveniently rewrite (5.34) as

$$
\left\{\begin{array}{rl}
\dot{\eta}_{2}= & \xi_{2}, \\
m\left(\eta_{2}, \xi_{2}\right) \dot{\xi}_{2}= & -1+2 a+\frac{1}{\beta}+\frac{\xi_{2}}{\beta \sqrt{\xi_{2}^{2}+4 d \beta}}-\frac{\tau \xi_{2}}{\sqrt{\left(\tau \xi_{2}\right)^{2}+4}} \\
& +\frac{1}{\beta}\left(1+\frac{\xi_{2}}{\sqrt{\xi_{2}^{2}+4 d \beta}}\right) \exp \left(-\eta_{2} \frac{\sqrt{\xi_{2}^{2}+4 d \beta}-\xi_{2}}{d}\right) \\
\equiv & f_{2}\left(\eta_{2}, \xi_{2} ; \tau\right) .
\end{array} \quad t>0\right.
$$

The nuclide of $f_{2}$ is drawn in Figs.5.7(a) and 5.8(a). Using the phase plane analysis for $(5.40)$, we arrive at the following result: Fix $\left(\eta_{2}(0), \xi_{2}(0)\right)$ to satisfy that $\eta_{2}(0)$ is very large and $\xi_{2}(0)$ takes nearly $-c_{3}$. (i) when $\tau$ is very small, then $\eta_{2}(t)$ is decreasing and $\eta_{2}\left(t_{1}\right)=0$ for some $t_{1}>0$, as in Fig.5.7(b); (ii) when $\tau>0$ is very close but less than $\tau_{c}$, then $\eta_{2}(t)$ decreases and change the direction and increases as if it were repulsive with the wall $x=0$, as in Fig.5.8(b).

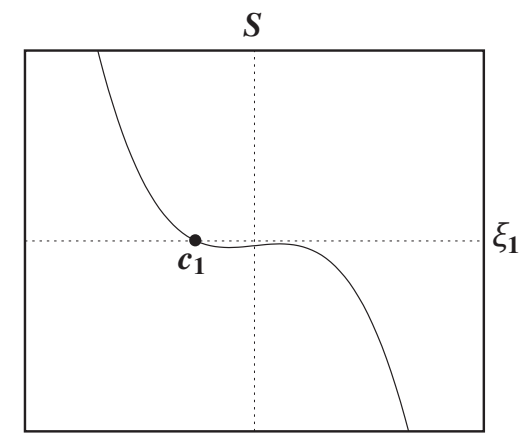

(a)

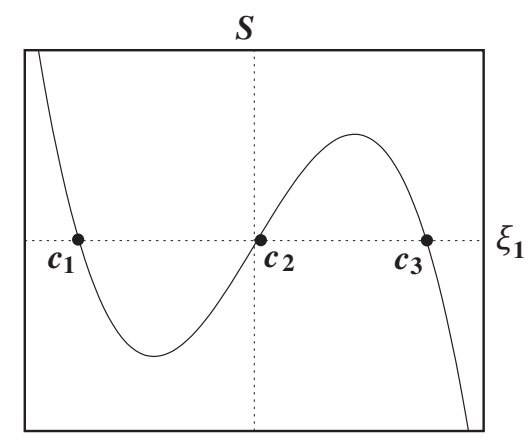

(b)

FIG. 5.6. Functions of $S\left(\xi_{1} ; \tau\right)$ with $\xi_{1}$ where $d=1.0, a=0.25, \gamma=0.9999999:(a) \tau=0.35355$; (b) $\tau=0.3535$.

Stage (ii): After Stage (i), one finds that $\eta_{1}(t)$ and $\eta_{2}(t)$ approach each other so as to satisfy $\eta_{1}(t)-\eta_{2}(t)=O(1)$. We therefore have to consider (5.27) for $\eta_{1}(t)$ and $\eta_{2}(t)$. Since Stage (i) indicates that $\eta_{1}(t)$ and $\eta_{2}(t)$ asymptotically moves with opposite velocities $c_{1}$ and $-c_{1}$, respectively, we may assume $\dot{\eta}_{1}(t)=-\dot{\eta}_{2}(t)$. Therefore, putting $2 \eta(t)=\eta_{1}(t)-\eta_{2}(t)$, (5.27) reduces to the following 2-dimensional ODEs for $\eta$ only:

$$
(\bar{m}(\dot{\eta})+\bar{n}(\eta, \dot{\eta})) \ddot{\eta}=1-2 a-\bar{v}(\eta, \dot{\eta})-\frac{\tau \dot{\eta}}{\sqrt{(\tau \dot{\eta})^{2}+4}}
$$

where

$$
\bar{n}(\eta, \dot{\eta})=\left(\frac{4 \eta^{2}}{\phi^{3}}+\frac{12 d \eta}{\phi^{4}}+\frac{12 d^{2}}{\phi^{5}}\right) \exp \left(-2 \eta \frac{\phi_{1-}}{2 d}\right)
$$




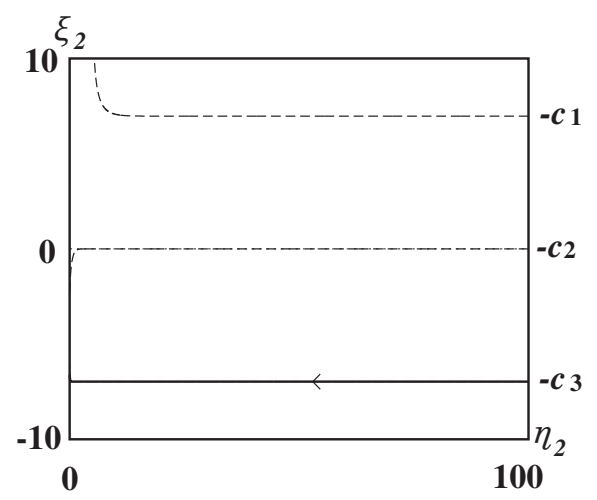

(a)

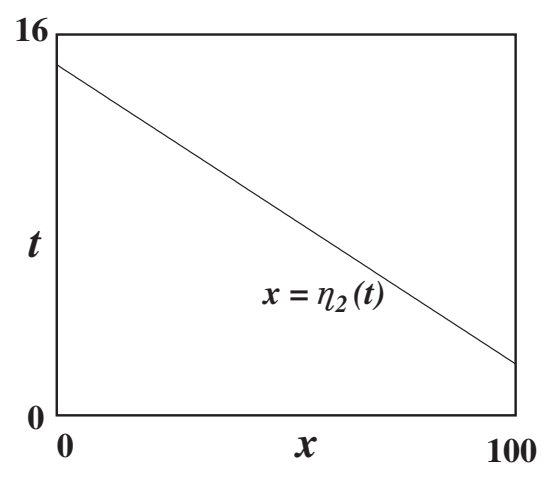

(b)

FIG. 5.7. Annihilation of the front interface where the parameters are the same as those in Fig.5.5 except $\tau=0.15$ : (a) Trajectory of (5.40) in the $\left(\eta_{2}, \xi_{2}\right)$-plane; (b) Time evolution of $\eta_{2}(t)$ of (5.40).

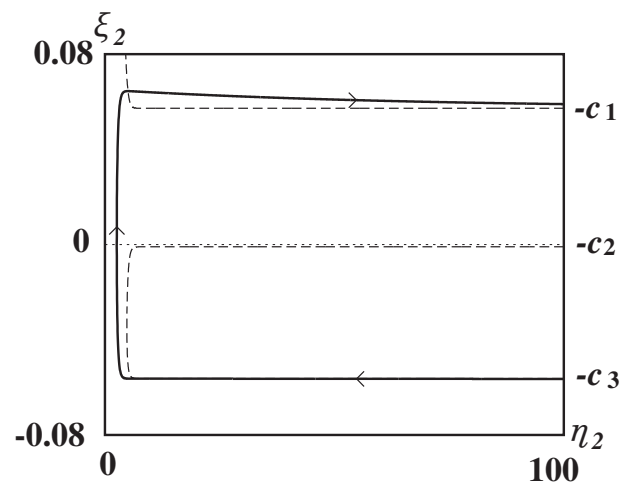

(a)

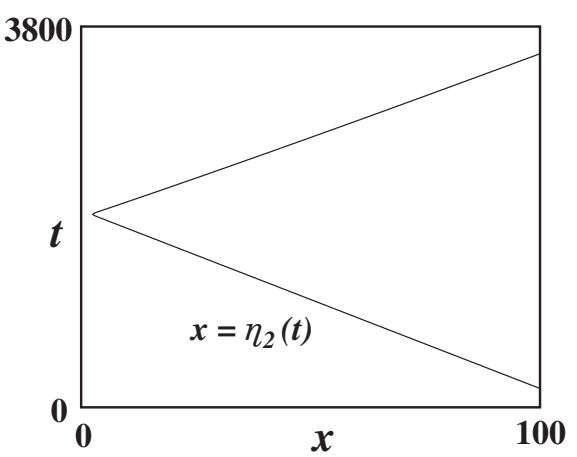

(b)

FIG. 5.8. Repulsion of the front interface where the parameters are the same as those in Fig.5.5 except $\tau=0.3535$ : (a) Trajectory of (5.40) in the $\left(\eta_{2}, \xi_{2}\right)$-plane; (b) Time evolution of $\eta_{2}(t)$ of (5.40).

and

$$
\bar{v}(\eta, \dot{\eta})=\frac{\phi_{-}}{\beta \phi}\left(1-\frac{\phi_{+}}{\beta \phi_{-}} \exp \left(-2 \eta \frac{\phi_{-}}{2 d}\right)\right) .
$$

Using the phase plane analysis in (5.41) again, it is found that $\eta_{1}(t)$ and $\eta_{2}(t)$ repel each other with the center $\left(\eta_{1}+\eta_{2}\right) / 2$ (Fig.5.9).

Stage (iii): Since this stage can be treated in a similar way to Stage (i), we omit the discussion.

6. Concluding remarks. In order to understand that very slowly travelling pulses repel each other, as if they are elastic objects, we have proposed a bistable RD system of the FitzHugh-Nagumo type and have used the interfacial dynamics approach to derive a limiting problem to describe the motion of interfaces from the RD system, 


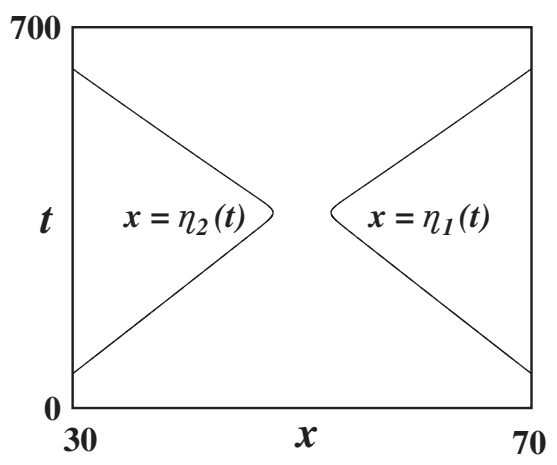

FIG. 5.9. Repulsion of the back interfaces. Time evolution of $\left(\eta_{1}(t), \eta_{2}(t)\right)$ of $(5.41)$ where the parameters are the same as those in Fig.5.5.

taking $\varepsilon \downarrow 0$. We thus obtained the fourth order system of ODEs to describe very slow motion of interfaces. Although the derivation is formal and has not yet been justified, the analysis with complementarily numerical calculations reveals that the reduced ODEs is a good approximate system to understand the repulsive interaction of very slowly travelling pulses. In a forthcoming paper, we consider the interaction of pulses, instead of interfaces, by using the pulse-dynamics methods and show that a general class of reaction-diffusion systems possess the pulse-reflection property, if the travelling velocity is sufficiently slow in a sense that travelling pulses bifurcate super-critically from a standing one.

Although, our conjecture is that all travelling pulses with very low velocity possess the repulsive property, it is not necessarily true that a travelling pulse with the repulsive property must have low velocity. Recently, it has been demonstrated in a suitable 3-component RD system that even if the velocity is not necessarily low, the travelling pulses reflect each other when they approach [9].

Acknowledgement. M.M. is supported by the Grant-in-Aid for Scientific Research (A) 12304006 and for Scientific Research of Priority Area (B)11214101. M.N. acknowledges the support of the Grant-in-Aid for Scientific Research 12740062. T.O. would like to thank the supports of the Grant-in-Aid for Scientific Research (B) 13554001 .

\section{Appendices.}

7.1. Appendix A. Although the derivation of (3.1)-(3.7) is already stated elsewhere (for instance, [1]), we show it. We formally put $\varepsilon=0$ in (2.1) and then obtain

$$
\begin{gathered}
v=f(u), \\
\frac{\partial v}{\partial t}=d \frac{\partial^{2} v}{\partial x^{2}}+ \begin{cases}h_{-}(v)-\gamma v, & t>0, \\
h_{+}(v)-\gamma v, & t>0, \quad x \in\left(\eta_{F}(t), \eta_{B}(t)\right),\end{cases}
\end{gathered}
$$

To determine the motion of the interface $x=\eta_{B}(t)$, for instance, let us introduce the new variable $\xi$ instead of $x$

$$
\xi=\frac{x-\eta_{B}(t)}{\varepsilon} \quad \text { for } \quad\left|x-\eta_{B}(t)\right|<\delta
$$


where $\delta=O(1)$. The resulting equations from (2.1) are given by

$$
\begin{gathered}
-\tau \dot{\eta_{B}}(t) \tilde{u}_{\xi}+\tau \varepsilon \tilde{u}_{t}=\tilde{u}_{\xi \xi}+f(\tilde{u})-\tilde{v}, \\
t>0, \quad-\delta / \varepsilon<\xi<\delta / \varepsilon, \\
-\varepsilon \dot{\eta_{B}}(t) \tilde{v}_{\xi}+\varepsilon^{2} \tilde{v}_{t}=d \tilde{v}_{\xi \xi}+\varepsilon^{2} g(\tilde{u}, \tilde{v}),
\end{gathered}
$$

where $\tilde{u}=u\left(\eta_{B}(t)+\varepsilon \xi, t\right), \tilde{v}=v\left(\eta_{B}(t)+\varepsilon \xi, t\right)$. In the limit $\varepsilon \downarrow 0$, (A.4) and (A.5) formally become

$$
\begin{gathered}
-\tau \dot{\eta}_{B}(t) \tilde{u}_{\xi}=\tilde{u}_{\xi \xi}+f(\tilde{u})-\tilde{v}, \\
t>0, \quad-\infty<\xi<+\infty, \\
d \tilde{v}_{\xi \xi}=0,
\end{gathered}
$$

where the boundary conditions are

$$
\begin{gathered}
\tilde{u}(t, \pm \infty)=h_{\mp}\left(v\left(t, \eta_{B}(t)\right)\right), \\
\tilde{v}(t, \pm \infty)=v\left(t, \eta_{B}(t)\right) .
\end{gathered}
$$

From (A.7) and (A.9), we obtain

$$
\tilde{v}(t, \xi)=v\left(t, \eta_{B}(t)\right) \text { for any } \xi \in \mathbf{R} \text {. }
$$

Therefore, (A.2) reduces to the following scalar equation for $\tilde{u}$ with the parameter $t$ :

$$
\begin{gathered}
-\tau \dot{\eta_{B}}(t) \tilde{u}_{\xi}=\tilde{u}_{\xi \xi}+f(\tilde{u})-v\left(t, \eta_{B}(t)\right), \\
\tilde{u}(t, \pm \infty)=h_{\mp}\left(v\left(t, \eta_{B}(t)\right)\right) .
\end{gathered}
$$

Solving the following nonlinear eigenvalue problem with $\lambda$

$$
\left\{\begin{array}{l}
-\lambda u_{z}=u_{z z}+f(u)-\xi, \quad z \in \mathbf{R}, \\
u(+\infty)=h_{-}(\xi), \quad u(-\infty)=h_{+}(\xi),
\end{array}\right.
$$

we obtain the interface equation for $\eta_{B}(t)$ as

$$
\tau \dot{\eta_{B}}(t)=\lambda\left(v\left(t, \eta_{B}(t)\right)\right) .
$$

By using a similar analysis to the above, the interface equation for $\eta_{F}(t)$ is also given by

$$
\tau \dot{\eta_{F}}(t)=-\lambda\left(v\left(t, \eta_{F}(t)\right)\right) .
$$


7.2. Appendix B. We derive the interface equations $(5.26) \mathrm{D}$ We extend $\mathbf{R}_{+}$to the whole domain $\mathbf{R}$, by using the mirror symmetry at $x=0$. Applying the Fourier transform given by

$$
v_{q}(t)=\int_{-\infty}^{\infty} v(x, t) e^{i q x} d x
$$

to (5.18), we have

$$
\dot{v}_{q}(t)=-\left(d q^{2}+\beta\right) v_{q}(t)+\frac{2}{q}\left(\sin \left(q \eta_{1}(t)\right)-\sin \left(q \eta_{2}(t)\right)\right.
$$

where the term $v_{q}(0) \exp \left(-\left(d q^{2}+\beta\right) t\right)$ was ignored, since we consider the dynamics after large time. The inverse Fourier transform of (B.1) yields

$$
\begin{aligned}
v(x, t)= & \frac{1}{2 \pi} \int_{-\infty}^{\infty} \frac{2}{q} \int_{0}^{t} \exp \left(-\left(d q^{2}+\beta\right)(t-s)-i q x\right) \sin \left(q \eta_{1}(s)\right) d s d q \\
& -\frac{1}{2 \pi} \int_{-\infty}^{\infty} \frac{2}{q} \int_{0}^{t} \exp \left(-\left(d q^{2}+\beta\right)(t-s)-i q x\right) \sin \left(q \eta_{2}(s)\right) d s d q
\end{aligned}
$$

Hence we obtain

$$
\begin{aligned}
v_{1}(t)= & v\left(\eta_{1}(t), t\right) \\
= & \frac{1}{2 \pi} \int_{-\infty}^{\infty} \frac{2}{q} \int_{0}^{t} \exp \left(-\left(d q^{2}+\beta\right)(t-s)-i q \eta_{1}(t)\right) \sin \left(q \eta_{1}(s)\right) d s d q \\
& -\frac{1}{2 \pi} \int_{-\infty}^{\infty} \frac{2}{q} \int_{0}^{t} \exp \left(-\left(d q^{2}+\beta\right)(t-s)-i q \eta_{1}(t)\right) \sin \left(q \eta_{2}(s)\right) d s d q
\end{aligned}
$$

and

$$
\begin{aligned}
v_{2}(t)= & v\left(\eta_{2}(t), t\right) \\
= & \frac{1}{2 \pi} \int_{-\infty}^{\infty} \frac{2}{q} \int_{0}^{t} \exp \left(-\left(d q^{2}+\beta\right)(t-s)-i q \eta_{2}(t)\right) \sin \left(q \eta_{1}(s)\right) d s d q \\
& -\frac{1}{2 \pi} \int_{-\infty}^{\infty} \frac{2}{q} \int_{0}^{t} \exp \left(-\left(d q^{2}+\beta\right)(t-s)-i q \eta_{2}(t)\right) \sin \left(q \eta_{2}(s)\right) d s d q .
\end{aligned}
$$

(B.3) and (B.4) indicate that there is a time-delayed interaction between two pulses mediated by $v$. Therefore, to solve (B.3) and (B.4), we take account of the effects of the time-delayed interaction perturbatively and employ the following approximation:

$$
\eta_{i}(s)=\eta_{i}(t)+\dot{\eta}_{i}(t)(s-t)+\ddot{\eta}_{i}(t) \frac{(s-t)^{2}}{2}+\cdots \quad(i=1,2)
$$

More precise explanation of (B.5) is stated in Appendix C. After straightforward calculation, we approximate (B.3) up to $O\left(\ddot{\eta}_{i}\right)$ as

$$
v_{1}(t)=v_{1}^{(1)}(t)+v_{1}^{(2)}(t),
$$


where the term of $O\left(e^{-\beta t}\right)$ was ignored, since we consider the dynamics after large time and

$$
\begin{aligned}
v_{1}^{(1)}(t)= & \frac{1}{2 \pi} \int_{-\infty}^{\infty} \frac{2}{2 i q}\left(\frac{1}{d q^{2}+\beta+i q \dot{\eta}_{1}}-\frac{\exp \left(-2 i q \eta_{1}\right)}{d q^{2}+\beta-i q \dot{\eta}_{1}}\right. \\
& \left.-\frac{\exp \left(-i q\left(\eta_{1}-\eta_{2}\right)\right)}{d q^{2}+\beta+i q \dot{\eta}_{2}}+\frac{\exp \left(-i q\left(\eta_{1}+\eta_{2}\right)\right)}{d q^{2}+\beta-i q \dot{\eta}_{2}}\right) d q \\
= & \frac{1}{2 \beta \phi_{1}}\left(\left(\phi_{1-}-\phi_{1+} \exp \left(-2 \eta_{1} \frac{\phi_{1-}}{2 d}\right)\right)\right. \\
& -\frac{1}{2 \beta \phi_{2}}\left(\phi_{2-} \exp \left(-\left(\eta_{1}-\eta_{2}\right) \frac{\phi_{2+}}{2 d}\right)-\phi_{2+} \exp \left(-\left(\eta_{1}+\eta_{2}\right) \frac{\phi_{2-}}{2 d}\right)\right) \\
\equiv & v_{2}\left(\eta_{1}, \eta_{2}, \dot{\eta}_{1}, \dot{\eta}_{2}\right) .
\end{aligned}
$$

By (B.3), the first order correction $v_{1}^{(2)}$ is given as

$$
\begin{aligned}
v_{1}^{(2)}(t)= & \frac{1}{2 \pi} \int_{-\infty}^{\infty} \ddot{\eta}_{1}\left(\frac{1}{\left(d q^{2}+\beta+i q \dot{\eta}_{1}\right)^{3}}+\frac{\exp \left(-2 i q \eta_{1}\right)}{\left(d q^{2}+\beta-i q \dot{\eta}_{1}\right)^{3}}\right) \\
& -\ddot{\eta}_{2}\left(\frac{\exp \left(-i q\left(\eta_{1}-\eta_{2}\right)\right)}{\left(d q^{2}+\beta+i q \dot{\eta}_{2}\right)^{3}}+\frac{\exp \left(-i q\left(\eta_{1}+\eta_{2}\right)\right)}{\left(d q^{2}+\beta-i q \dot{\eta}_{2}\right)^{3}}\right) d q .
\end{aligned}
$$

We may derive the equation for $v_{2}$ in the similar way to (B.7) so that $v_{2}^{(1)}$ is given as

$$
\begin{aligned}
v_{2}^{(1)}(t)= & \frac{1}{2 \pi} \int_{-\infty}^{\infty} \frac{2}{2 i q}\left(-\frac{1}{d q^{2}+\beta+i q \dot{\eta_{2}}}+\frac{\exp \left(-2 i q \eta_{2}\right)}{d q^{2}+\beta-i q \dot{\eta_{2}}}\right. \\
& \left.+\frac{\exp \left(i q\left(\eta_{1}-\eta_{2}\right)\right)}{d q^{2}+\beta+i q \dot{\eta}_{1}}-\frac{\exp \left(-i q\left(\eta_{1}+\eta_{2}\right)\right)}{d q^{2}+\beta-i q \dot{\eta}_{1}}\right) d q \\
= & \frac{1}{2 \beta \phi_{2}}\left(\phi_{2+}+\phi_{2+} \exp \left(-2 \eta_{2} \frac{\phi_{2-}}{2 d}\right)\right) \\
& -\frac{1}{2 \beta \phi_{1}}\left(\phi_{1+} \exp \left(-\left(\eta_{1}-\eta_{2}\right) \frac{\phi_{1-}}{2 d}\right)+\phi_{1+} \exp \left(-\left(\eta_{1}+\eta_{2}\right) \frac{\phi_{-1}}{2 d}\right)\right) \\
\equiv & v_{2}\left(\eta_{1}, \eta_{2}, \dot{\eta}_{1}, \dot{\eta}_{2}\right) .
\end{aligned}
$$

By (B.4), the correction $v_{2}^{(2)}$ is obtained as

$$
\begin{aligned}
-v_{2}^{(2)}(t)= & \frac{1}{2 \pi} \int_{-\infty}^{\infty} \ddot{\eta_{2}}\left(\frac{1}{\left(d q^{2}+\beta+i q \dot{\eta}_{2}\right)^{3}}+\frac{\exp \left(-2 i q \eta_{2}\right)}{\left(d q^{2}+\beta-i q \dot{\eta}_{2}\right)^{3}}\right) \\
& -\ddot{\eta}_{1}\left(\frac{\exp \left(i q\left(\eta_{1}-\eta_{2}\right)\right)}{\left(d q^{2}+\beta+i q \dot{\eta}_{1}\right)^{3}}+\frac{\exp \left(-i q\left(\eta_{1}+\eta_{2}\right)\right)}{\left(d q^{2}+\beta-i q \dot{\eta}_{1}\right)^{3}}\right) d q .
\end{aligned}
$$

Thus one may write $v_{1}^{(2)}$ and $v_{2}^{(2)}$ as

$$
v_{1}^{(2)}=m\left(\eta_{1}, \dot{\eta}_{1}\right) \ddot{\eta_{1}}-n_{1}\left(\eta_{1}, \eta_{2}, \dot{\eta}_{2}\right) \ddot{\eta_{2}}
$$


and

$$
-v_{2}^{(2)}=m\left(\eta_{2}, \dot{\eta_{2}}\right) \ddot{\eta_{2}}-n_{2}\left(\eta_{1}, \eta_{2}, \dot{\eta_{1}}\right) \ddot{\eta_{1}}
$$

where $m_{i} \quad(i=1,2)$ are defined by

$$
\begin{aligned}
m_{i}\left(\eta_{i}, \dot{\eta}_{i}\right) & =\frac{1}{2 \pi} \int_{-\infty}^{\infty}\left(\frac{1}{\left(d q^{2}+\beta+i q \dot{\eta}_{i}\right)^{3}}+\frac{\exp \left(-2 i q \eta_{i}\right)}{\left(d q^{2}+\beta-i q \dot{\eta}_{i}\right)^{3}}\right) d q \\
& =\frac{6 d^{2}}{\phi_{i}^{5 / 2}}\left(1+\exp \left(-2 \eta_{i} \phi_{i-}\right)\right)+\left(\frac{2 \eta_{i}^{2}}{\phi_{i}^{3 / 2}}+\frac{6 d \eta_{i}}{\phi_{i}^{2}}\right) \exp \left(-2 \eta_{i} \phi_{i-}\right) .
\end{aligned}
$$

In the similar way to (B.12), $n_{1}$ and $n_{2}$ are defined by

$$
\begin{aligned}
& n_{1}=\frac{1}{2 \pi} \int_{-\infty}^{\infty}\left(\frac{\exp \left(-i q\left(\eta_{1}-\eta_{2}\right)\right)}{\left(d q^{2}+\beta+i q \dot{\eta}_{2}\right)^{3}}+\frac{\exp \left(-i q\left(\eta_{1}+\eta_{2}\right)\right)}{\left(d q^{2}+\beta-i q \dot{\eta}_{2}\right)^{3}}\right) d q \\
& =\left(\frac{\left(\eta_{1}-\eta_{2}\right)^{2}}{2 \phi_{2}^{3 / 2}}+\frac{3 d\left(\eta_{1}-\eta_{2}\right)}{\phi_{2}^{2}}+\frac{6 d^{2}}{\phi_{2}^{5 / 2}}\right) \exp \left(-\left(\eta_{1}-\eta_{2}\right) \phi_{2+}\right) \\
& +\left(\frac{\left(\eta_{1}+\eta_{2}\right)^{2}}{2 \phi_{2}^{3 / 2}}+\frac{3 d\left(\eta_{1}+\eta_{2}\right)}{\phi_{2}^{2}}+\frac{6 d^{2}}{\phi_{2}^{5 / 2}}\right) \exp \left(-\left(\eta_{1}+\eta_{2}\right) \phi_{2-}\right) \\
& \equiv n_{1}\left(\eta_{1}, \eta_{2}, \dot{\eta}_{2}\right) \text {, } \\
& n_{2}=\frac{1}{2 \pi} \int_{-\infty}^{\infty}\left(\frac{\exp \left(i q\left(\eta_{1}-\eta_{2}\right)\right)}{\left(d q^{2}+\beta+i q \dot{\eta}_{1}\right)^{3}}+\frac{\exp \left(-i q\left(\eta_{1}+\eta_{2}\right)\right)}{\left(d q^{2}+\beta-i q \dot{\eta}_{1}\right)^{3}}\right) d q \\
& =\left(\frac{\left(\eta_{1}-\eta_{2}\right)^{2}}{2 \phi_{1}^{3 / 2}}+\frac{3 d\left(\eta_{1}-\eta_{2}\right)}{\phi_{1}^{2}}+\frac{6 d^{2}}{\phi_{1}^{5 / 2}}\right) \exp \left(-\left(\eta_{1}-\eta_{2}\right) \phi_{1-}\right) \\
& +\left(\frac{\left(\eta_{1}+\eta_{2}\right)^{2}}{2 \phi_{1}^{3 / 2}}+\frac{3 d\left(\eta_{1}+\eta_{2}\right)}{\phi_{1}^{2}}+\frac{6 d^{2}}{\phi_{1}^{5 / 2}}\right) \exp \left(-\left(\eta_{1}+\eta_{2}\right) \phi_{1-}\right) \\
& \equiv n_{2}\left(\eta_{1}, \eta_{2}, \dot{\eta}_{1}\right) .
\end{aligned}
$$

Therefore (5.26) can be obtained.

7.3. Appendix C. In this appendix, we examine the condition that the approximation (5.25) holds. The main part is to clarify the reason why one may ignore the higher order time derivative in the pulse equation of motion. We will show below that this approximation is justified in the vicinity of the supercritical pulse-bifurcation point $\tau_{*}$.

It is pointed out that there is another bifurcation threshold $\tau_{c}$ for the front motion. Actually, the expansion in terms of $c_{1}=\dot{\eta}_{1}$ of the right hand side of (5.26) becomes

$$
\begin{aligned}
m \dot{c_{1}} & -n \dot{c_{2}}+\left(\tau-\tau_{c}\right) c_{1}+h(\tau) c_{1}^{3}=\alpha \\
& +\frac{1}{\beta}\left(\exp \left(-2 \eta_{1} \sqrt{\beta / d}\right)+\exp \left(-\left(\eta_{1}-\eta_{2}\right) \sqrt{\beta / d}\right)-\exp \left(-\left(\eta_{1}+\eta_{2}\right) \sqrt{\beta / d}\right)\right)
\end{aligned}
$$


where $\alpha=1-2 a-1 / \beta, \tau_{c}=1 / \beta \sqrt{d \beta}$ and

$$
h(\tau)=\frac{1}{16 d \beta^{2} \sqrt{d \beta}}-\frac{\tau^{3}}{16}
$$

and a similar equation for $c_{2}=\dot{\eta_{2}}$. The coefficient $h(\tau)$ is positive for $\tau \sim \tau_{c}$.

As shown just below (5.15), the stationary solution is $c_{1}=O\left(\delta^{1 / 2}\right)$ where $\delta=$ $\sqrt{\tau_{*}-\tau}$. Here we have used the fact that $\tau_{*}-\tau_{c}=O\left(\delta^{3 / 2} \log \delta\right)$ which will be shown below in (C.5). Therefore it is convenient to introduce the scaled velocity

$$
\hat{c_{1}}=c_{1} / \delta^{1 / 2} \text {. }
$$

Since the motion of a pulse is very slow near the bifurcation threshold, we make a scaling of time as

$$
\hat{t}=\delta t
$$

In terms of these scaled quantities, all the terms on the left hand side of (C.1) are found to be of order of $\delta^{3 / 2}$. The higher order time-derivative ignored in (C.1) can be written as

$$
\frac{d^{n} c_{1}}{d t^{n}}=\delta^{1 / 2+n} \frac{d^{n} \hat{c_{1}}}{d \hat{t}^{n}}
$$

Therefore these derivatives for $n \geq 2$ are higher order in $\delta$.

To balance all the terms in (C.1), the constant $\alpha$ should be regarded as $\alpha=$ $O\left(\delta^{3 / 2}\right)$ and the front and back positions are also restricted to satisfy this smallness. The difference $\tau_{*}-\tau_{c}$ is given from (5.12) and (5.16) by

$$
\tau_{*}-\tau_{c}=\frac{\alpha \tau_{c}}{\beta}\left(1+s_{0} \sqrt{\frac{\beta}{d}}\right) .
$$

This indicates $\tau_{*}-\tau_{c}=O\left(\delta^{3 / 2} \log \delta\right)$.

\section{REFERENCES}

[1] P.C.FIfE, Dynamics of internal layers and diffusive interfaces, CBMS-NSF Regional Conference Series in Applied Mathematics 53 (Philadelphia: SIAM, 1980).

[2] P.Gray And S.K.SCOTT, Autocatalytic reaction in the isothermal continuous stirred tank reactor, Chem. Engng. Sci., 39 (1984), pp. 1087-1097.

[3] D.Hilhost, Y.Nishiura and M.Mimura, shape A free boundary problem arising in some reacting-diffusing system, Proc. Roy. Soc. Edinburgh, 118A (1991), pp. 355-378.

[4] H.IKEDA, Singular pulse wave bifurcations from front and back waves in bistable reactiondiffusion systems, Methods and Applications of Analysis, 3 (3) (1996), pp. 285-317.

[5] H.IKEDA, Existence and stability of pulse waves bifurcated from front and back waves in bistable reaction-diffusion, Japan J. Indust. Appl. Math., 15 (1998), pp. 163-231.

[6] H.IKEDA AND T.IKEDA, Bifurcation phenomena from standing pulse solutions in some reactiondiffusion systems, J.Dynamics and Differential Equations, 12 (2000), pp. 117-167.

[7] T.IKedA, H.IKEDA AND M.Mimura, Hopf bifurcation of travelling pulses in some bistable reaction-diffusion systems, Methods and Applications of Analysis, 7 (2000), pp. 165-194.

[8] S.KAWAGUChI AND M.Mimura, Collision of travelling waves in a reaction-diffusion system with global coupling effect, SIAM J. Appl. Math., 59 (1999), pp. 920-941.

[9] S.Kawaguchi And M.Mimura, Synergistic effect of two inhibitors and one activator in a reaction-diffusion system, manuscript.

[10] K.Krisher AND A.MiKailov, Bifurcation to travelling spots in reaction-diffusion systems, Phys. Rev. Lett., 73 (1994), pp. 3165-3168. 
[11] M.Mimura And M.Nagayama, Non-annihilation dynamics in an exothermic reaction-diffusion system with mono-stable excitability, CHAOS, 7 (4) (1997), pp. 817-826.

[12] J.Nagumo, S.Arimoto and S.Yoshizawa, An active pulse transmission line simulating nerve axon, Proc. I. R. E., 50 (1962), pp. 2061-2070.

[13] T.OHTA AND J.Kiyose, Collision of domain boundaries in a reaction-diffusion system, J. Phys. Soc. Jpn., 65 (1996), pp. 1967-1970.

[14] T.OHta, J.Kiyose And M.Mimura, Collision of propagating pulse in a reaction-diffusion system, J. Phys. Soc. Jpn., 66 (1997), pp. 1551-1558.

[15] J.Pearson, Complex patterns in a simple system, Sciences, 261 (1993), pp. 189-192.

[16] V.Petrov, S.ScotT AND K.Showalter, Excitability, wave reflection, and wave splitting in a cubic autocatalysis reaction-diffusion systems, Phil. Trans. R. Sco. Lond., A347 (1994), pp. $631-642$.

[17] J.Rinzel AND D.Terman, Propagation phenomena in a bistable reaction-diffusion system, SIAM J. Appl. Math., 42 (1982), pp. 1111-1137.

[18] J.J.TYSON AND J.P.KEENER, Singular perturbation theory of travelling waves in excitable media, Physica D, 32 (1988), pp. 327-361.

[19] X.-F.CHEN, Generation and propagation of interfaces in reaction-diffusion systems, Trans. Math. Soc., 334 (1992), pp. 877-913. 
